\title{
Pneumatosis intestinalis versus pseudo-pneumatosis: review of CT findings and differentiation
}

\author{
Jin Hong Wang • Alessandro Furlan • Diana Kaya • \\ Satoshi Goshima $\cdot$ Mitchell Tublin $\cdot$ Kyongtae T. Bae
}

Received: 7 September 2010 /Accepted: 18 November 2010 /Published online: 21 December 2010

(C) European Society of Radiology 2010

\begin{abstract}
Pneumatosis intestinalis is defined as the presence of gas within the wall of the gastrointestinal tract. Originally described on plain abdominal radiographs, it is an imaging sign rather than a specific diagnosis and it is associated with both benign and lifethreatening clinical conditions. The most common lifethreatening cause of pneumatosis intestinalis is bowel ischaemia. Computed tomography (CT) is usually requested to detect underlying disease. The presence of pneumatosis intestinalis often leads physicians to make a diagnosis of serious disease. However, an erroneous diagnosis of pneumatosis intestinalis may be made (i.e. pseudo-pneumatosis) when intraluminal beads of gas are trapped within or between faeces and adjacent mucosal folds. The purpose of this pictorial essay is to review and describe the CT imaging findings of pneumatosis and pseudo-pneumatosis intestinalis and to discuss key discriminatory imaging features.
\end{abstract}

This pictorial essay was presented and awarded as an educational exhibit at RSNA 2008

\footnotetext{
J. H. Wang $\cdot$ A. Furlan $\cdot$ D. Kaya $\cdot$ M. Tublin $\cdot$ K. T. Bae $(\bowtie)$ Department of Radiology,

University of Pittsburgh School of Medicine,

Presbyterian South Tower, 200 Lothrop St,

Pittsburgh, PA 15213, USA

e-mail: baek@upmc.edu

S. Goshima

Department of Radiology, Gifu University School of Medicine, Yanagido,

Gifu 501-1193, Japan
}

Keywords Computed tomography Intramural gas . Pneumatosis intestinalis · Pseudo-pneumatosis intestinalis . Differentiation

\section{Introduction}

Pneumatosis intestinalis is defined as the presence of gas within the wall of the gastrointestinal tract. It is an imaging sign rather than a specific diagnosis [1]. The gas in pneumatosis intestinalis may originate from the gastrointestinal lumen, bacterial production, or from the lungs [2]. The causes of pneumatosis intestinalis are traditionally divided into two categories: benign conditions (such as asthma, scleroderma and pyloric stenosis) and life-threatening conditions (such as bowel obstruction, necrotising enterocolitis, bowel ischaemia, cancer, trauma and drugs) [1,3]. The most common life-threatening cause of pneumatosis intestinalis is bowel ischaemia [1]. Computed tomography (CT) is usually requested to detect underlying disease. The presence of pneumatosis intestinalis often leads physicians to make a diagnosis of serious disease [3]. However, an erroneous diagnosis of pneumatosis intestinalis may be made (i.e. pseudo-pneumatosis) when intraluminal beads of gas are trapped within or between faeces and the adjacent mucosal folds (Fig. 1). Awareness of features that may differentiate between pneumatosis and pseudo-pneumatosis - an incidental imaging diagnosis - is critical for appropriate clinical management [4]. In this article, we review and describe the CT findings of both pneumatosis intestinalis and pseudopneumatosis, and provide key imaging findings that can help differentiate pseudo-pneumatosis from true pneumatosis. 


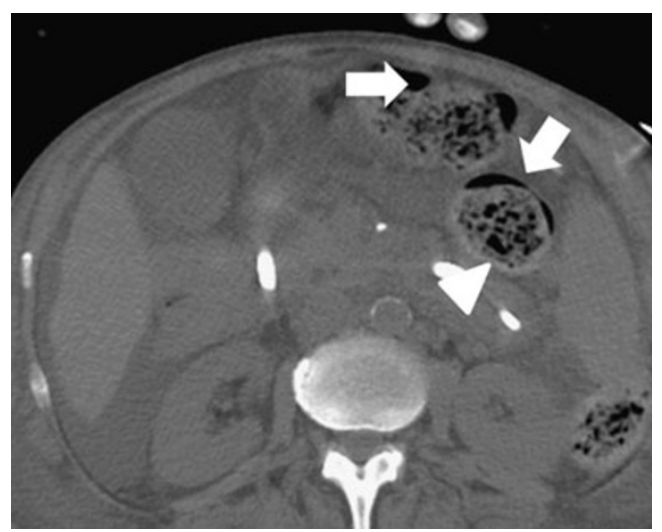

Fig. 1 Pseudo-pneumatosis in a 52-year-old man with abdominal distension. Axial unenhanced CT shows gas collections within faeces and between faeces and bowel wall (arrows), consistent with pseudopneumatosis. Note the thin layer of high-attenuation enteric contrast surrounding the stool (arrowhead) simulating intestinal wall. This might lead to a misdiagnosis of true pneumatosis

\section{Imaging techniques}

Abdominal radiography and $\mathrm{CT}$ are the most common imaging techniques used to diagnose pneumatosis intestinalis. However, abdominal radiography is often too insensitive to detect early stages of pneumatosis or assess the underlying disease $[2,5]$. CT is the best imaging investigation for the diagnosis of pneumatosis intestinalis (Figs. 2, 3) because of its high sensitivity for detecting even small intramural gas collections $[2,6]$. The administration of oral and intravenous contrast media in $\mathrm{CT}$ is not required for the diagnosis of pneumatosis but may be useful in delineating the bowel lumen and wall for the confirmation

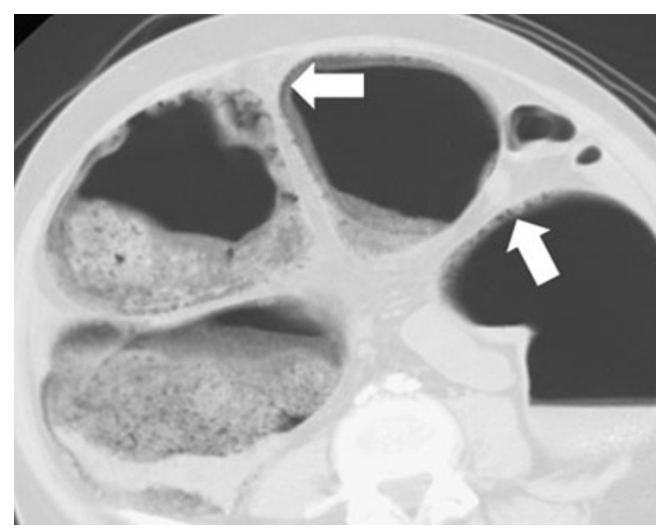

Fig. 2 Extensive ischaemic colon in a 63-year-old woman post-surgical resection for rectal carcinoma. Axial CT at a lung window shows massively dilated colon with diffuse wall thickening and intramural gas (arrows), consistent with pneumatosis intestinalis. The patient was found to have extensive ischaemic colon at emergency laparotomy

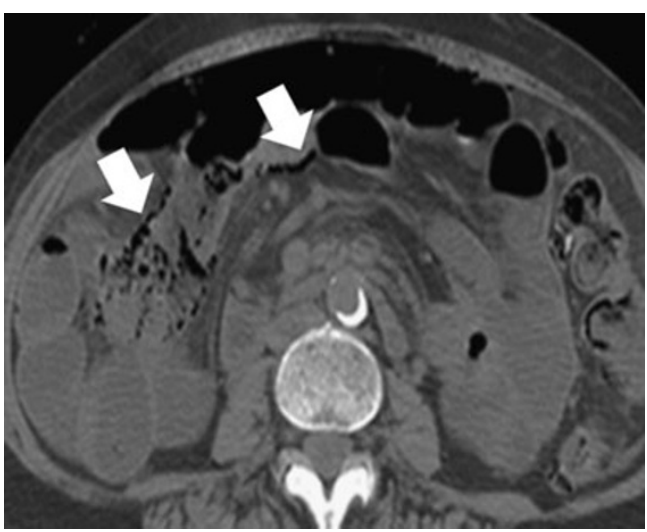

Fig. 3 Pneumatosis intestinalis in a 58-year-old man with small bowel obstruction secondary to adhesion at the distal ileum. Axial unenhanced CT shows diffusely dilated small bowel with pneumatosis intestinalis (arrows) in the jejunum

or exclusion of other diagnoses. Representation of CT images at multiplanar (i.e. coronal, sagittal) reformats may help determine the location and the extent of gas within the intestinal wall (Fig. 4). Finally, while abdominal CT images are routinely displayed in a soft tissue window, use of the lung window is advantageous for the detection of subtle intraluminal gas (Fig. 4) [4].

\section{Imaging findings of pneumatosis and pseudo-pneumatosis intestinalis}

\section{Pneumatosis intestinalis}

Radiolucency within the bowel wall (Fig. 5) is the hallmark of pneumatosis on abdominal radiography [2]. The patterns of pneumatosis shown at CT may vary considerablylinear, bubble, curvilinear gas collections (Fig. 6); gas may be localised or diffuse throughout the gastrointestinal tract [7]. Gas patterns were reported to be useful to differentiate benign and clinically worrisome pneumatosis intestinalis [4, 6]. In addition, other $\mathrm{CT}$ findings such as soft-tissue thickening of the bowel wall, free fluid and peri-intestinal soft-tissue stranding are more frequently associated with clinically worrisome than benign pneumatosis intestinalis [8]. Nonetheless, the severity of the clinical manifestations usually correlates with the underlying disease rather than the extent or pattern of gas [8-10].

Intramural gas may extend beyond the bowel wall into portomesenteric veins, particularly in the setting of bowel ischaemia [11, 12]. On CT images, portomesenteric venous gas is typically visualised as tubular or branched mesenteric lucencies and linear, branched gas collections within the periphery of the liver (Fig. 7). Mesenteric venous gas can be distinguished from pneumoperitoneum by its tubular 

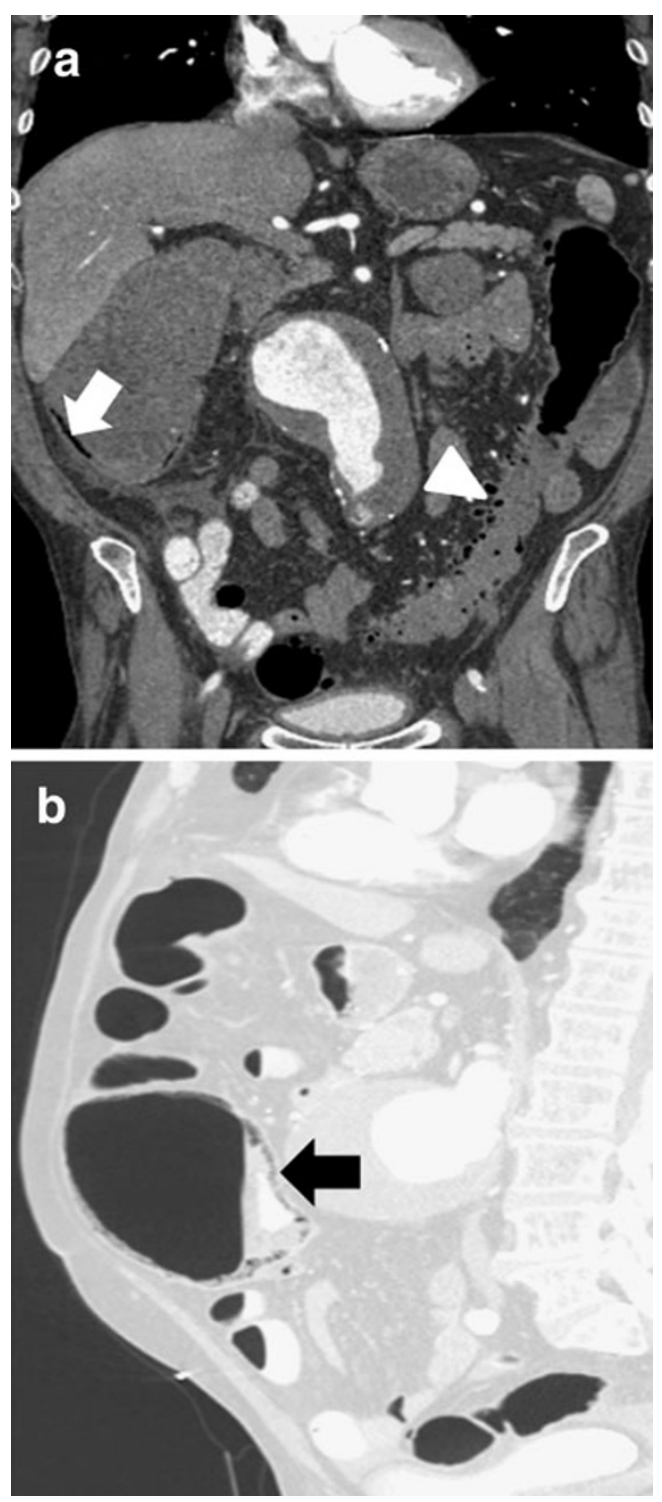

Fig. 4 Intramural gas of the colon in a 73-year-old man with worsening abdominal pain. a Coronal contrast-enhanced CT shows dilated right colon with intramural versus intraluminal gas (arrow), aortic aneurysm and decompressed descending colon with extensive sigmoid diverticulosis (arrowhead). b Sagittal contrast-enhanced CT displayed in a lung window confirms the intramural location of the gas collection (arrow), consistent with pneumatosis intestinalis, extending beyond the free gas-fluid level within the bowel lumen

appearance and its location within mesenteric venous branches (Fig. 8) [13]. Furthermore, intrahepatic portal venous gas appears with tubular lucencies in the periphery of the liver, whereas pneumobilia is usually distributed centrally [4]. Whether the presence of portomesenteric venous gas can predict the severity of ischaemia remains a controversial issue. Several studies reported a higher mortality rate with the combination of pneumatosis and portal venous gas $[3,14]$, while others $[10,15,16]$

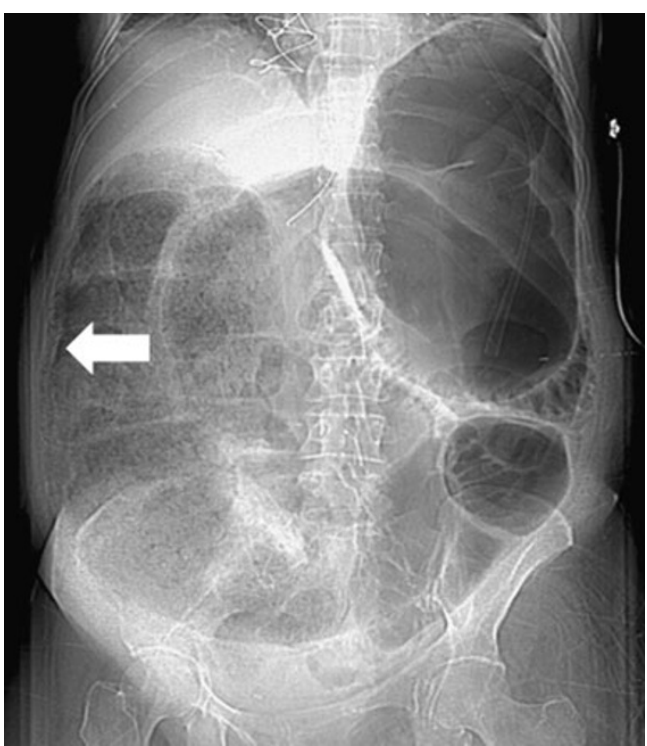

Fig. 5 Intramural gas in a 63-year-old woman post-surgical resection for rectal carcinoma. Abdominal radiograph shows diffuse lucency within colonic wall (arrow)

suggested only a weak correlation between the two. The underlying pathophysiology of portomesenteric venous gas is still unclear; suggested causes include intestinal wall alterations, bowel distension and sepsis $[1,13,16]$.

Besides intramural gas and its extension, observation on bowel wall alterations could be helpful in defining pneumatosis intestinalis [14]. The intramural gas could go within the circumference of the bowel wall, which may extend superior to a luminal gas-fluid level (Fig. 9). The intramural gas could dissect within the bowel wall edge

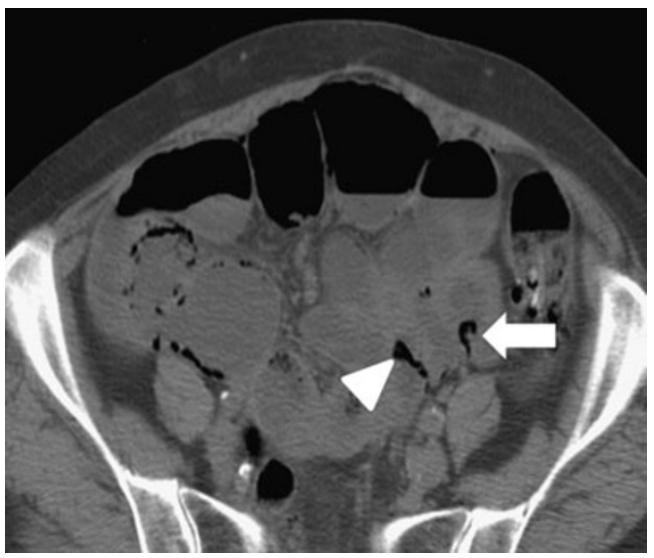

Fig. 6 Intramural gas in a different shape in a 66-year-old man with scleroderma. Axial unenhanced CT shows dilated small bowel with curvilinear-like (arrow), bubble-like (arrowhead) intramural gas, suggestive of pneumatosis intestinalis, that was not identified at follow-up CT (not shown) 
Fig. 7 Intramural and portomensenteric venous gas in a 66-year-old asymptomatic man after duodenal ulcer repair. a Axial unenhanced CT shows diffuse, bubble-like and curvilinear intramural gas collections (arrows) throughout the small bowel, consistent with pneumatosis intestinalis. b, c Axial CT inal levels show diffuse branching lucency in the mesenteric vessels (arrow, b) and the periphery of the liver indicating portal vein gas (arrow, c). d Axial unenhanced $\mathrm{CT}$, acquired at the same level as a after 10 days of follow-up, shows complete resolution of pneumatosis intestinalis following a benign clinical course images at two different abdom-
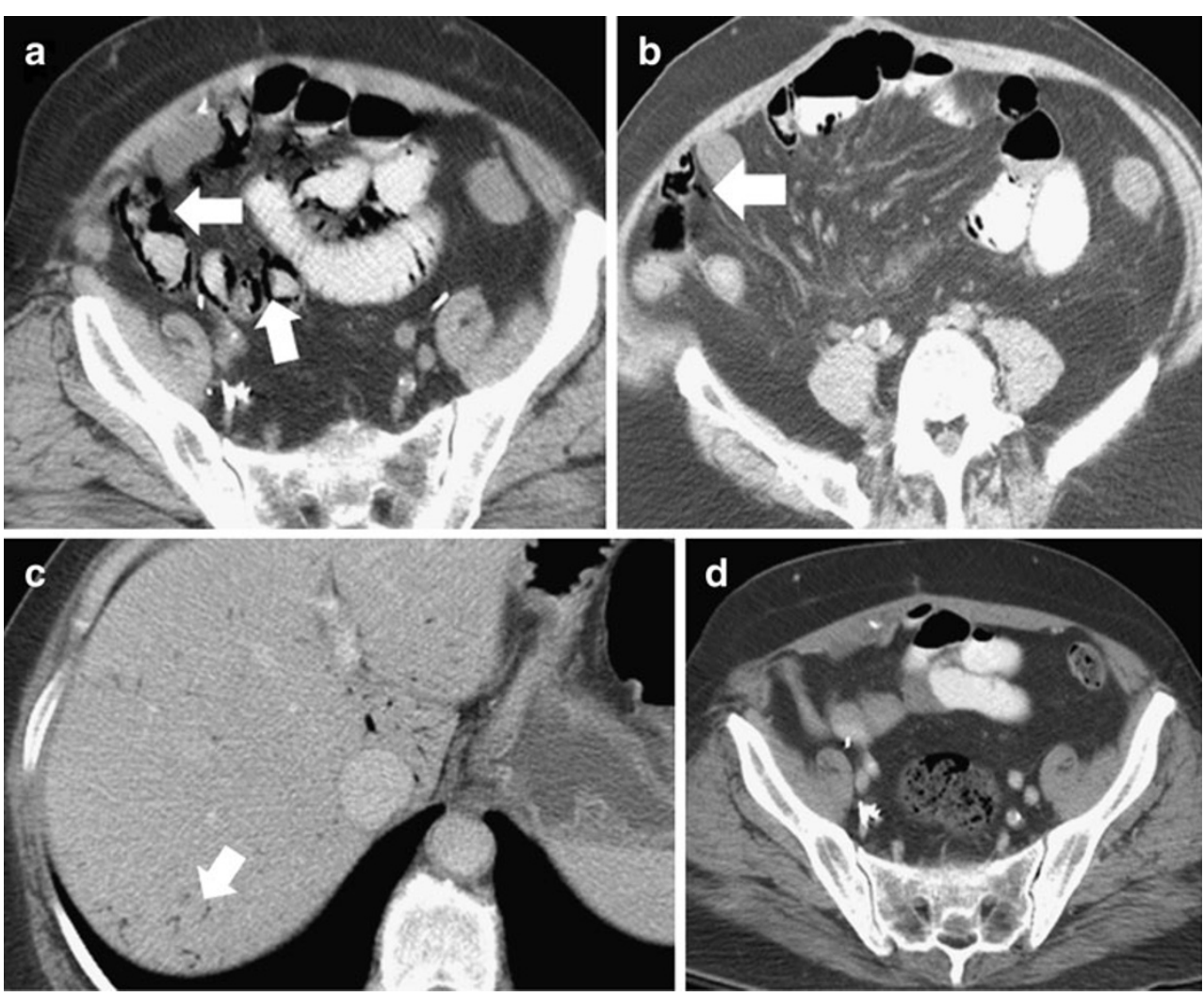

Fig. 8 Intrahepatic and tubular gas in a venous branch in a 45year-old woman with superior mesenteric artery thrombosis (SMA). a Sagittal contrastenhanced CT shows SMA thrombosis (arrow). b Axial contrast-enhanced CT shows intramural gas of pneumatosis intestinalis (arrow). c Axial CT (lung window) shows tubular lucency in a venous branch (arrow), consistent with mesenteric venous gas. d Axial contrast-enhanced CT shows extensive intrahepatic portal venous gas (arrow)
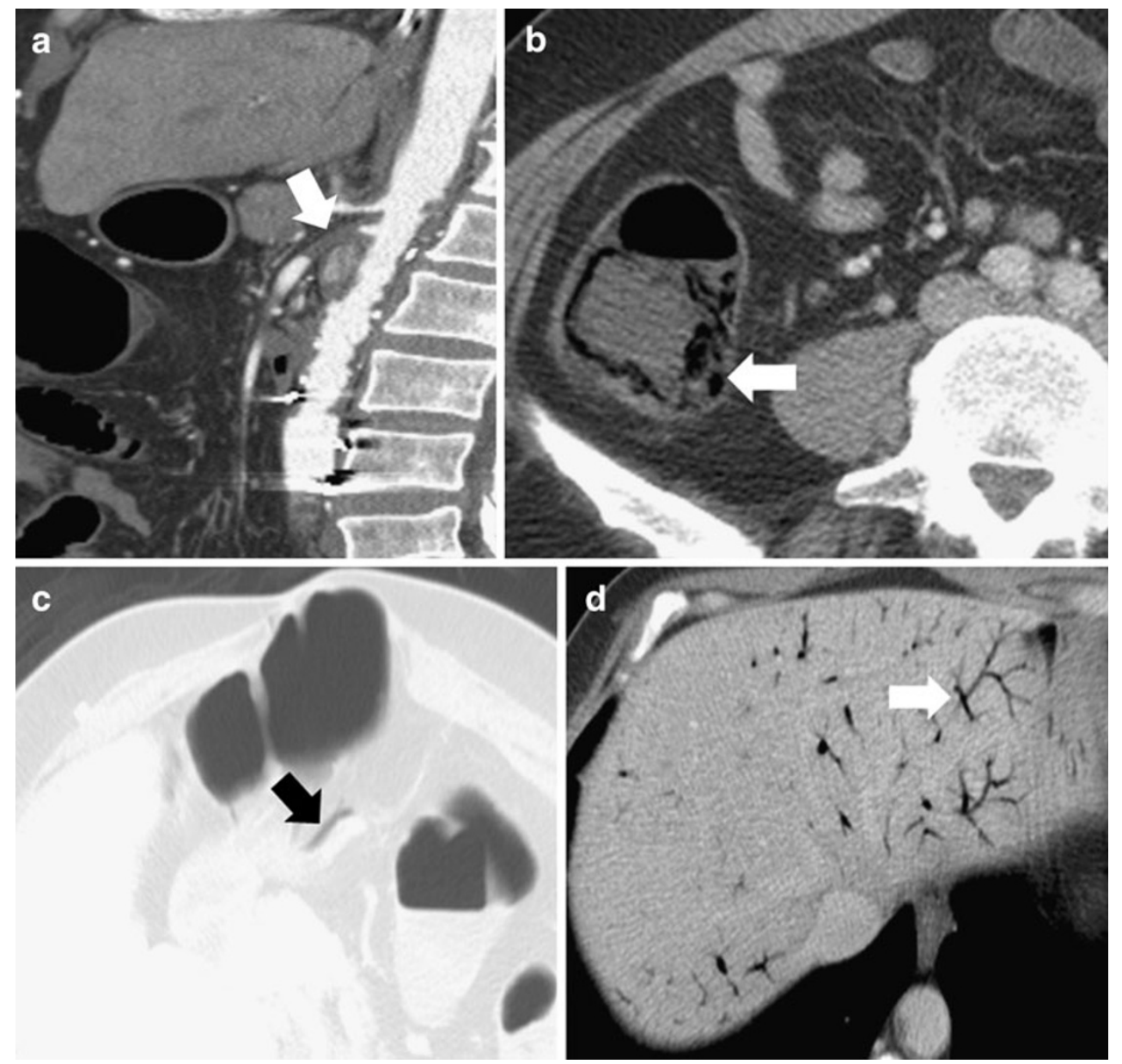


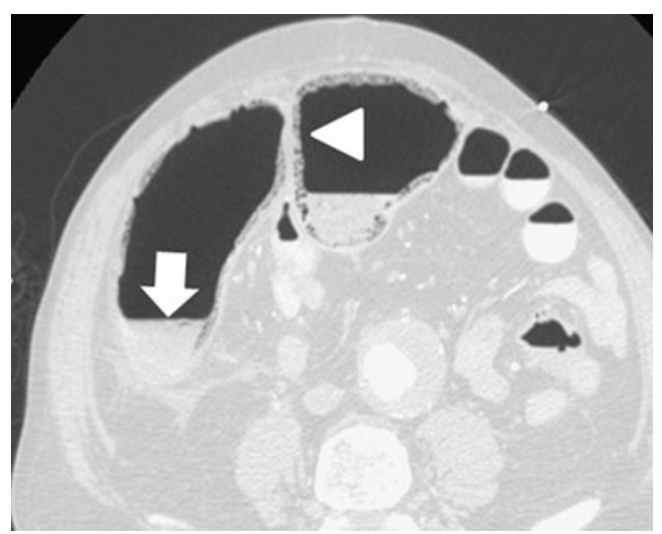

Fig. 9 Intramural gas in a 73-year-old man with worsening abdominal pain. Axial contrast-enhanced CT shows gas within the circumference of the bowel wall (arrowhead) which extends superior to a luminal gas-fluid level (arrow), indicating pneumatosis intestinalis

(Fig. 10). Both findings may reflect transmural damage of the bowel wall. In addition, a relatively smooth contiguous intraluminal gas column could outline the smooth bowel wall (Fig. 11). The relatively smooth gas column against the bowel wall may be a sign of extensive swelling of the bowel wall.

Although pneumatosis intestinalis is an important sign representing a pathological state of the bowel, a clinical management decision for pneumatosis intestinalis should be ultimately made on the basis of multiple factors, including clinical symptoms, physical examination findings and laboratory test results [8]. Other CT findings suggestive of predisposing diseases include dilatation of the bowel from a bowel obstruction, mesenteric oedema and bowel wall thickening related to ischaemia, a concomitant mass and pneumoperitoneum (Fig. 12). It should be noted, however, that pneumoperitoneum may be also caused by relatively

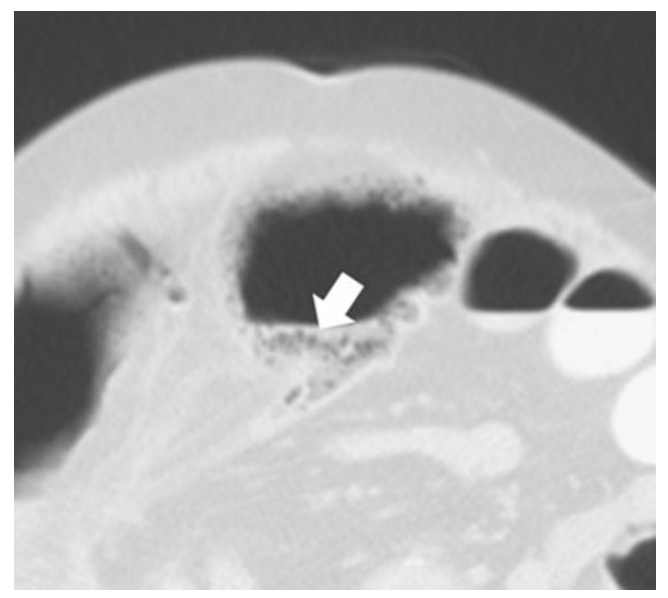

Fig. 10 Intramural gas in a 71-year-old man with abdominal pain. Axial contrast-enhanced CT shows gas dissecting within the bowel wall edge (arrow), consistent with pneumatosis intestinalis

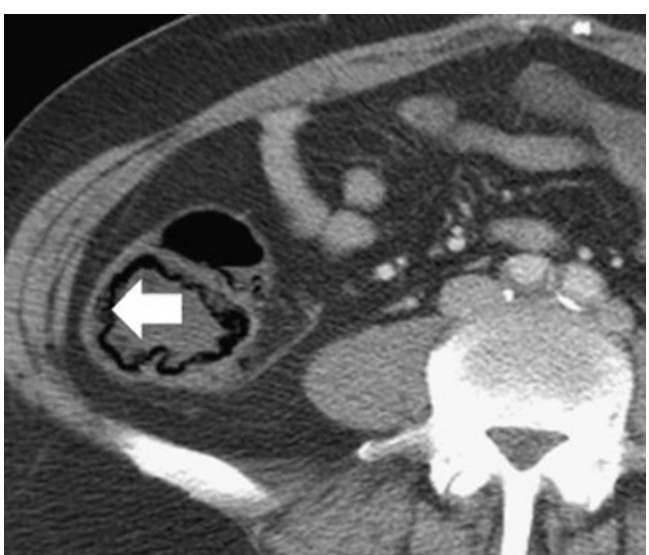

Fig. 11 Smooth intraluminal gas column outlining the bowel wall in a 45-year-old woman with superior mesenteric artery thrombosis (SMA). Axial unenhanced CT shows a relatively smooth, contiguous gas column (arrow), more commonly seen in pneumatosis intestinalis

benign conditions such as a rupture of subserosal cysts in benign primary pneumatosis and connective tissue diseases (Fig. 13) [4].

\section{Pseudo-pneumatosis intestinalis}

Gas trapped within faeces or against the mucosal surface may mimic the intramural gas of pneumatosis intestinalis. Although this so-called "pseudo-pneumatosis" may sometimes be indistinguishable from true pneumatosis, the appearance and distribution of the gas collections, normal bowel wall as well as additional CT imaging findings may help differentiate between clinically relevant pneumatosis and incidental pseudo-pneumatosis.

\section{Differentiations between pneumatosis and pseudo-pneumatosis}

Patients with either pseudo-pneumatosis or benign causes of pneumatosis may be asymptomatic or present with mildto-moderate symptoms such as shortness of breath, chronic abdominal pain and constipation [17]. Patients may have a history of steroid use, chemotherapy and organ transplantation. Patients with life-threatening causes of pneumatosis may present with moderate to severe abdominal symptoms such as diarrhoea, bloody stools, abdominal distension or severe abdominal pain. The physical findings are usually unremarkable $[4,8,18]$.

CT findings useful for differentiating pneumatosis (intramural gas) versus pseudo-pneumatosis (intra-luminal gas) include the location, distribution and pattern of gas. Pneumatosis intestinalis occurs focally or throughout the small bowel or colon [19]. Pseudo-pneumatosis is more 

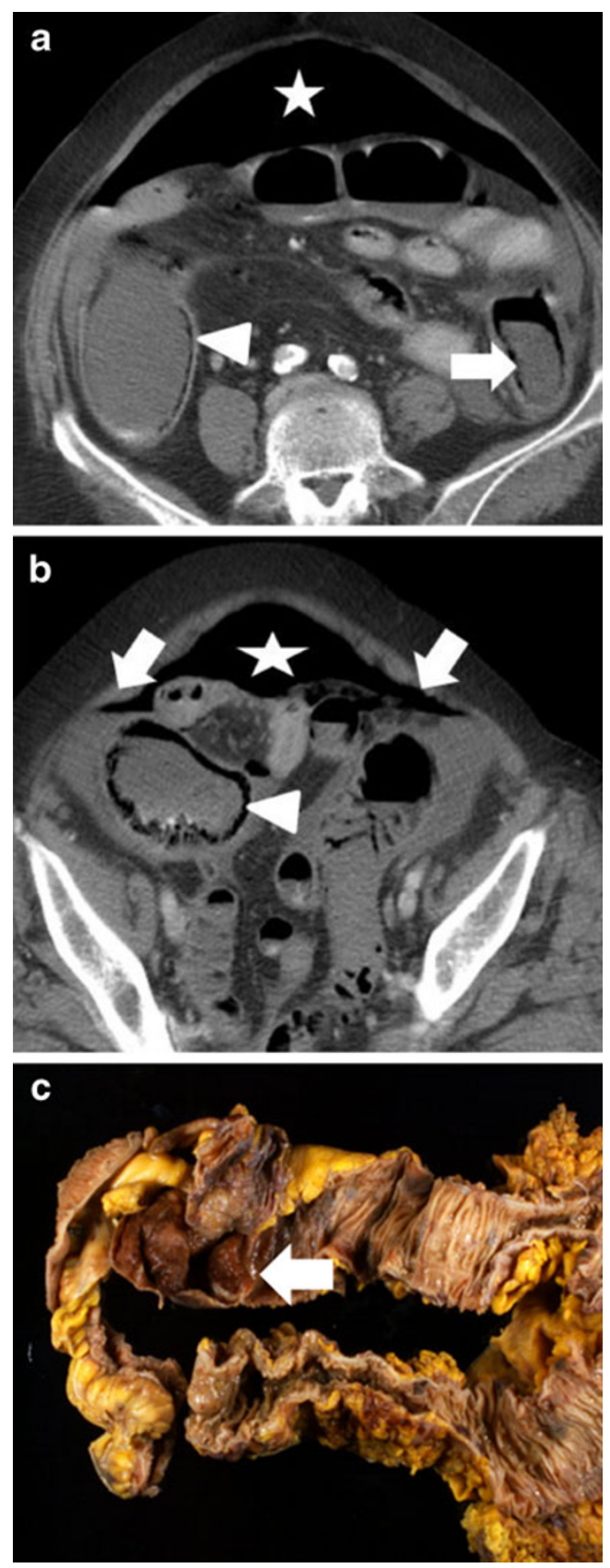

Fig. 12 Intramural gas and pneumoperitoneum in an 80-year-old woman with worsening abdominal pain. a, b Axial unenhanced CT show intramural gas (arrowhead) in the colon, consistent with pneumatosis intestinalis, thickened descending colonic wall (short arrow, a), extensive pneumoperitoneum (star, a and b), and extraluminal contrast medium leak (arrows, b). c Gross pathological specimen after emergency surgery shows ischaemic colitis and wall perforation (arrow)

frequently seen in the caecum and ascending colon (because of an admixture of liquid stool and gas). The distribution of gas at the interface of the intestinal wall, which may reflect bowel wall alterations, is useful for differentiating pneumatosis from pseudo-pneumatosis [13].
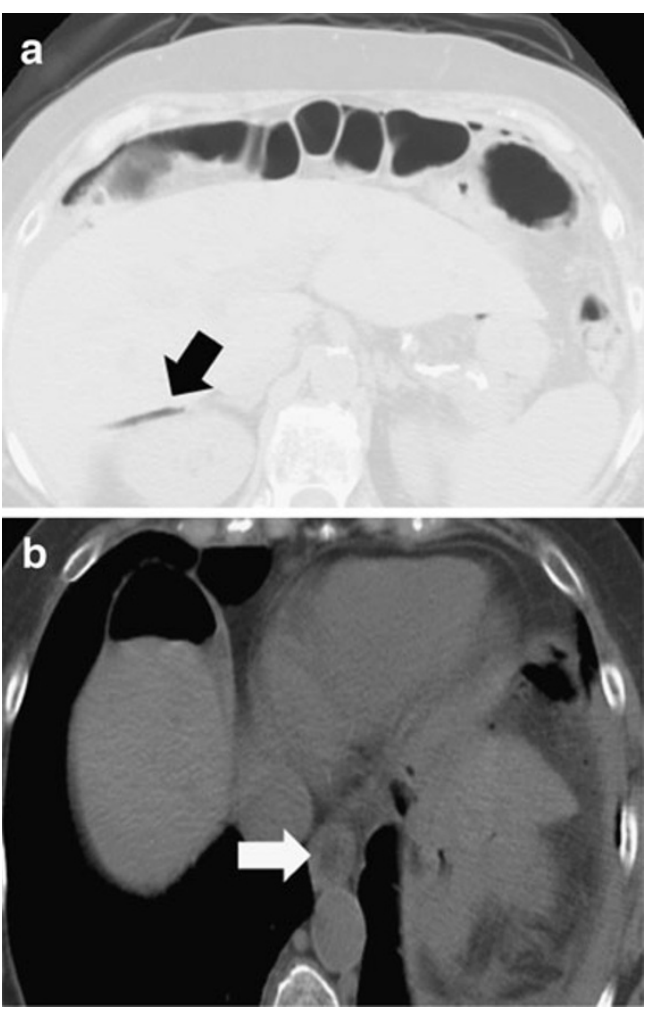

Fig. 13 Pneumoperitoneum in a 66-year-old man with a history of scleroderma and multiple episodes of pneumatosis intestinalis spontaneously resolved in $24 \mathrm{~h}$. a Axial unenhanced CT at a lung window shows pneumoperitoneum (arrow). b Axial unenhanced CT shows oesophageal dilation (arrow), indicating patulous oesophagus associated with scleroderma

For example, a gas collection against the bowel wall that stops at the free gas-fluid level within the bowel lumen suggests pseudo-pneumatosis (Fig. 14), whereas gas along the bowel wall beyond the gas-fluid level is more

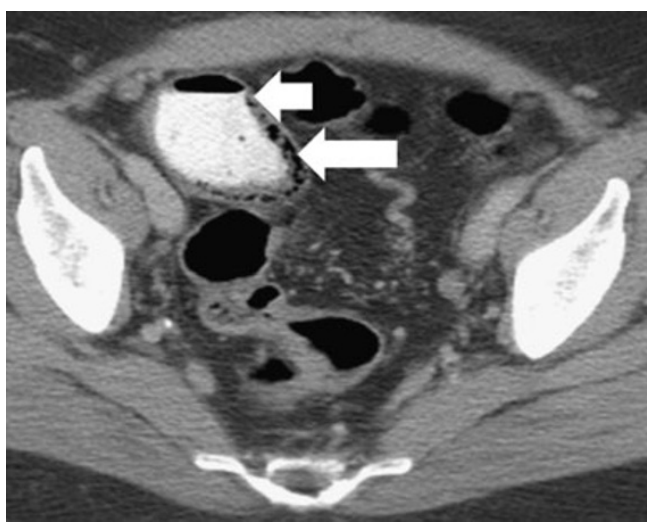

Fig. 14 Pseudo-pneumatosis intestinalis in a 50-year-old woman with abdominal pain. Axial contrast-enhanced CT shows irregular punctuated gas column (long arrow) between the faeces and mucosal folds, which stops at the free gas-fluid level (short arrow), consistent with pseudo-pneumatosis intestinalis 

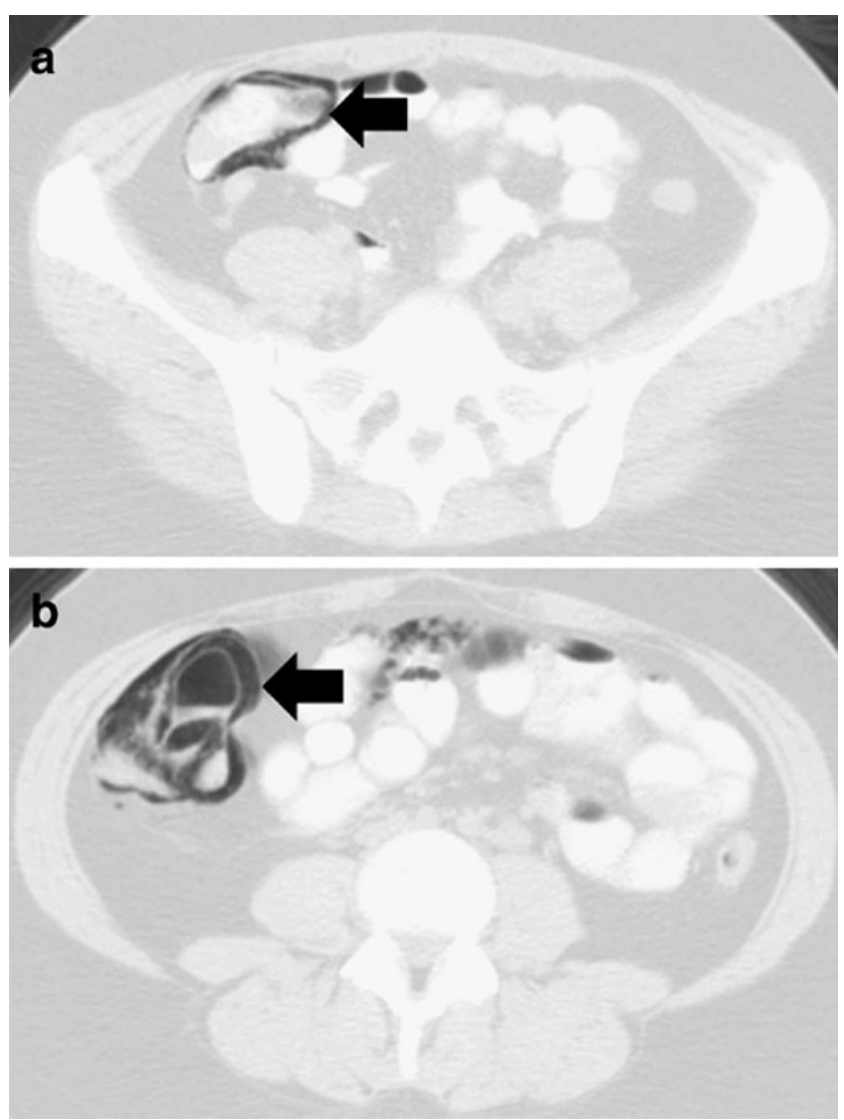

Fig. 15 Intramural gas in a 26-year-old woman with worsening abdominal pain. a Axial CT at a lung window shows a relatively smooth, contiguous gas column (arrow). b Axial CT at a lung window at a slightly different level from a shows intramural gas beyond the gas-fluid level (arrow), indicating pneumatosis intestinalis

consistent with pneumatosis intestinalis (Fig. 15). The pattern of gas may also be a helpful discriminator. An irregularly punctuated gas column pattern (from intact mucosal folds) is more commonly seen with pseudo-

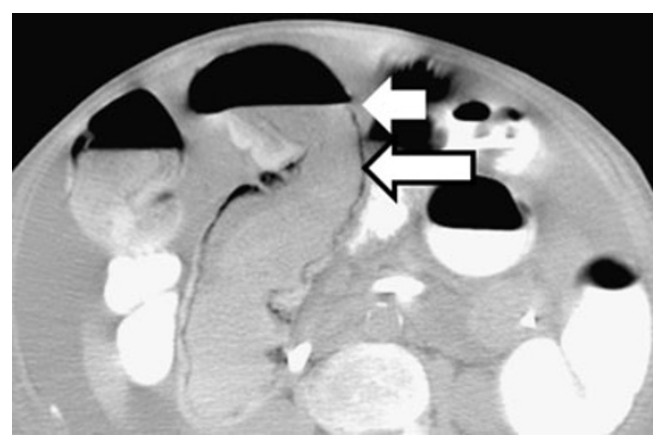

Fig. 16 Pseudo-pneumatosis intestinalis in a 63-year-old woman with treated rectal cancer. Axial contrast-enhanced CT shows an irregular column of gas (arrow) between the wall and the intestinal content stopping at the free gas-fluid level (short arrow) within the bowel lumen, consistent with pseudo-pneumatosis intestinalis pneumatosis intestinalis (Fig. 16), while a relatively smooth contiguous gas column pattern (reflecting oedematous bowel wall) is suggestive of pneumatosis intestinalis. Finally, the presence of portomesenteric venous gas and additional findings such as vascular embolisation and cancer can be important signs indicative of the presence of pneumatosis intestinalis.

\section{Conclusion}

In conclusion, portal or mesenteric venous gas, intramural gas superior to a gas-fluid level, continuous gas outlining the bowel wall and dissecting gas in the bowel wall edge are features characteristic of pneumatosis. Conversely, these findings are absent in pseudo-pneumatosis. Gas confined to the inner wall of the caecum/proximal right colon which terminates at a gas-fluid level is strongly suggestive of pseudo-pneumatosis. Knowledge of these key CT imaging findings may prevent an erroneous diagnosis of potentially life-threatening bowel ischaemia.

\section{References}

1. Pear BL (1998) Pneumatosis intestinalis: a review. Radiology 207:13-19

2. St Peter SD, Abbas MA, Kelly KA (2003) The spectrum of pneumatosis intestinalis. Arch Surg 138:68-75

3. Morris MS, Gee AC, Cho SD, Limbaugh K, Underwood S, Ham B, Schreiber MA (2008) Management and outcome of pneumatosis intestinalis. Am J Surg 195:679-682, discussion 682-673

4. Ho LM, Paulson EK, Thompson WM (2007) Pneumatosis intestinalis in the adult: benign to life-threatening causes. AJR Am J Roentgenol 188:1604-1613

5. Lund EC, Han SY, Holley HC, Berland LL (1988) Intestinal ischemia: comparison of plain radiographic and computed tomographic findings. Radiographics 8:1083-1108

6. Soyer P, Martin-Grivaud S, Boudiaf M, Malzy P, Duchat F, Hamzi Z, Pocard M, Vahedi K, Rymer R (2008) Linear or bubbly: a pictorial review of $\mathrm{CT}$ features of intestinal pneumatosis in adults. J Radiol 89:1907-1920

7. Thoeni RF, Cello JP (2006) CT imaging of colitis. Radiology 240:623-638

8. Olson DE, Kim YW, Ying J, Donnelly LF (2009) CT predictors for differentiating benign and clinically worrisome pneumatosis intestinalis in children beyond the neonatal period. Radiology 253:513-519

9. Feczko PJ, Mezwa DG, Farah MC, White BD (1992) Clinical significance of pneumatosis of the bowel wall. Radiographics 12:1069-1078

10. Wiesner W, Mortele KJ, Glickman JN, Ji H, Ros PR (2001) Pneumatosis intestinalis and portomesenteric venous gas in intestinal ischemia: correlation of CT findings with severity of ischemia and clinical outcome. AJR Am J Roentgenol 177:1319-1323

11. Schindera ST, Triller J, Vock P, Hoppe H (2006) Detection of hepatic portal venous gas: its clinical impact and outcome. Emerg Radiol 12:164-170 
12. Wiesner W, Mortele KJ, Glickman JN, Ji H, Ros PR (2002) Portal-venous gas unrelated to mesenteric ischemia. Eur Radiol 12:1432-1437

13. Sebastia C, Quiroga S, Espin E, Boye R, Alvarez-Castells A, Armengol M (2000) Portomesenteric vein gas: pathologic mechanisms, CT findings, and prognosis. Radiographics 20:12131224, discussion 1224-1216

14. Kernagis LY, Levine MS, Jacobs JE (2003) Pneumatosis intestinalis in patients with ischemia: correlation of CT findings with viability of the bowel. AJR Am J Roentgenol 180:733-736

15. Faberman RS, Mayo-Smith WW (1997) Outcome of 17 patients with portal venous gas detected by CT. AJR Am J Roentgenol 169:1535-1538
16. Hou SK, Chern CH, How CK, Chen JD, Wang LM, Lee CH (2004) Hepatic portal venous gas: clinical significance of computed tomography findings. Am J Emerg Med 22:214-218

17. Pickhardt PJ, Kim DH, Taylor AJ (2008) Asymptomatic pneumatosis at CT colonography: a benign self-limited imaging finding distinct from perforation. AJR Am J Roentgenol 190:W112W117

18. Lassandro F, di Santo Stefano ML, Maria Porto A, Grassi R, Scaglione M, Rotondo A (2010) Intestinal pneumatosis in adults: diagnostic and prognostic value. Emerg Radiol 17(5):361-365

19. Federle MPJ, Jeffrey RB, Desser TS, Anne VS, Eraso A, Chen J, Chabra S, Pealer KM (2004) Diagnostic imaging - Abdomen. In: Pneumatosis of the intestine. Amirsys, Salt Lake City, pp 34-37 